\title{
Lowered serum cesium levels in schizophrenia: association with immune-inflammatory biomarkers and cognitive impairments
}

\author{
Abbas F. Almulla, ${ }^{1}$ Shatha R. Moustafa, ${ }^{2}$ Arafat H. Al-Dujaili, ${ }^{3}$ Hussein K. Al-Hakeim, ${ }^{4}$ \\ Michael Maes ${ }^{5,6,7}$ iD \\ ${ }^{1}$ Medical Laboratory Technology Department, College of Medical Technology, The Islamic University, Najaf, Iraq. ${ }^{2}$ Clinical Analysis \\ Department, College of Pharmacy, Hawler Medical University, Havalan City, Erbil, Iraq. ${ }^{3}$ Senior Clinical Psychiatrist at the Faculty of Medicine, \\ University of Kufa, Najaf, Iraq. ${ }^{4}$ Department of Chemistry, College of Science, University of Kufa, Najaf, Iraq. ${ }^{5}$ Department of Psychiatry, \\ Faculty of Medicine, Chulalongkorn University, Bangkok, Thailand. ${ }^{6}$ Department of Psychiatry, Medical University of Plovdiv, Plovdiv, Bulgaria. \\ ${ }^{7}$ IMPACT Strategic Research Centre, Deakin University, Geelong, VIC, Australia.
}

\begin{abstract}
Objectives: A previous study has shown that schizophrenia (SCZ) is accompanied by lowered levels of trace/metal elements, including cesium. However, it is not clear whether changes in cesium, rubidium, and rhenium are associated with activated immune-inflammatory pathways, cognitive impairments, and the symptomatology of SCZ.

Methods: This study measured cesium, rubidium, and rhenium, cognitive impairments (using the Brief Assessment of Cognition in Schizophrenia [BACS]), and the levels of cytokines/chemokines interleukin (IL)-1 $\beta$, tumor necrosis factor (TNF)- $\alpha$, and eotaxin (CCL11) in 120 patients with SCZ and 54 healthy controls. Severity of illness was assessed using the Brief Psychiatric Rating Scale (BPRS), the Scale for the Assessment of Negative Symptoms (SANS), the Fibromyalgia and Chronic Fatigue Syndrome Rating (FF) Scale, and the Hamilton Depression Rating Scale (HAM-D).

Results: Serum cesium was significantly lower in patients with SCZ as compared with controls. Further, serum cesium was significantly and inversely associated with CCL11 and TNF- $\alpha$, but not IL-1 $\beta$, in patients with SCZ; significant inverse associations were also noted between serum cesium levels and BPRS, FF, HAM-D, and SANS scores. Finally, cesium was positively correlated with neurocognitive probe results including the Tower of London, Symbol Coding, Controlled Word Association, Category Instances, Digit Sequencing Task, and List Learning tests.

Conclusion: The results suggest that lowered serum cesium levels may play a role in the pathophysiology of SCZ, contributing to specific symptom domains including negative, depressive and fatigue symptoms, neurocognitive impairments (spatial working, episodic, and semantic memory and executive functions), and neuroimmune pathways.
\end{abstract}

Keywords: Inflammation; neuroimmunomodulation; major depression; chronic fatigue syndrome; myalgic encephalomyelitis; biomarkers

\section{Introduction}

Schizophrenia (SCZ), a severe and debilitating mental illness affecting about $1 \%$ of the world population, is characterized by negative and positive symptoms and neurocognitive impairments. ${ }^{1}$ A biological predisposition with multi-genetic risk factors, changes in neurotransmitter systems, and neuroimmune factors - and psychosocial stress are associated with increased risk of SCZ. ${ }^{2}$ The first comprehensive neuroimmune theory of SCZ was introduced in 1995 by Smith \& Maes, ${ }^{3}$ who, considering that $T$ lymphocytes and activated macrophages are key phenomena in the pathophysiology of SCZ, proposed the

Correspondence: Michael Maes, Department of Psychiatry, Faculty of Medicine, Chulalongkorn University, Patumwan, 10330, Bangkok, Thailand.

E-mail: dr.michaelmaes@ @otmail.com

Submitted Feb 14 2020, accepted Apr 09 2020, Epub Jun 122020. "macrophage-lymphocyte theory." Since then, many studies have confirmed that SCZ is accompanied by activation of the immune-inflammatory response system (IRS) and the compensatory immune-regulatory system (CIRS). ${ }^{4-6}$ SCZ is also accompanied by changes in inflammatory biomarkers, including increased levels of neurotoxic cytokines and chemokines such as interleukin (IL)-1 $\beta$, tumor necrosis factor (TNF)- $\alpha$, and eotaxin (CCL11). ${ }^{4-6}$ Furthermore, recent studies have described attenuated levels of antioxidants, ${ }^{7}$ including enzymatic antioxidant components/molecules and some trace elements. ${ }^{8-10}$ These immune and oxidative stress biomarkers are significantly associated with positive, negative, and
How to cite this article: Almulla AF, Moustafa SR, Al-Dujaili $A H$, Al-Hakeim HK, Maes M. Lowered serum cesium levels in schizophrenia: association with immune-inflammatory biomarkers and cognitive impairments. Braz J Psychiatry. 2021;43:131-137. http:// dx.doi.org/10.1590/1516-4446-2020-0908 
neurocognitive SCZ symptoms, such as deficits in semantic and episodic memory and executive functions.,

Some investigators have recently reported that SCZ is accompanied by changes in trace/metal elements, although contradictory results were obtained with different types of metals, SCZ, and methods. ${ }^{11-15}$ Importantly, Cai et al. ${ }^{15}$ found a significant reduction in serum levels of the alkali metal cesium in individuals with SCZ in comparison with healthy controls. This may be important because cesium shares some similarities with potassium, and therefore may interfere with potassium metabolism, ${ }^{16}$ while a single nucleotide polymorphism (SNP) in the $K C N H 2$ gene, which encodes the pore-forming subunit of potassium channels, has been associated with SCZ and with cognitive impairments. ${ }^{17}$ Moreover, cesium, rhenium, and rubidium were previously assayed in sera of patients with another immune-inflammatory illness, namely rheumatoid arthritis, with cesium found to be decreased in patients as compared with healthy controls. ${ }^{18}$ Furthermore, cesium may inhibit GSK3 $\beta$ and attenuate oxidative stress-induced cell death and neuronal apoptotic pathways. ${ }^{19}$ In mice, a central nervous system (CNS)-active carborane containing cesium has antidepressant effects through inhibition of pore formation by the cation-selective purinergic receptor ion channel. ${ }^{20}$

Like potassium, rubidium is also found in high levels in red blood cells, viscera, and muscle tissues. ${ }^{21}$ Rubidium shares some chemical properties with potassium, and may replace potassium in the $\mathrm{Na}^{+}-\mathrm{K}^{+}$-ATPase system; in turn, $\mathrm{K}^{+}$-dependent ATPase is activated by low rubidium concentrations. ${ }^{22}$ In addition, rubidium may stimulate human metabolism due to its chemical similarities to $\mathrm{K}^{+} .{ }^{21}$ Rhenium does not have known biological functions, although rhenium-containing compounds are highly toxic. ${ }^{23}$ Only few studies have studied these elements in SCZ, even though cesium and rubidium play a role in biological systems, and despite the known association of cesium with immune-inflammatory conditions.

Thus, the present study measured cesium, rhenium, and rubidium in patients with SCZ in order the estimate the associations between these metals and immune biomarkers (IL-1 $\beta$, TNF- $\alpha$, and CCL11), the symptom subdomains of SCZ (including depression), and neurocognitive impairments.

\section{Methods}

\section{Participants}

This study included 120 patients with SCZ and 54 healthy controls recruited at the Ibn-Rushd Training Hospital for Psychiatric Medicine, Baghdad, Iraq (December 2018 until February 2019). The 54 apparently healthy controls were staff members or their family members and friends of patients with SCZ. Patients with SCZ were in a stabilized phase of illness and did not show acute psychotic episodes for at least 1 year prior to the study. DSM-IV-TR criteria were used to diagnose SCZ. ${ }^{24}$ Healthy controls were excluded if they had a lifetime or current DSM-IV-TR axis I disorder or a positive family history of psychosis. Individuals with SCZ were excluded if they had acute psychotic episodes the year prior to the study or axis-1 DSM-IV-TR disorders other than SCZ, including bipolar disorder, major depression, schizoaffective disorder, obsessive-compulsive disorder (OCD), psycho-organic disorders, and substance use disorders. All subjects had C-reactive protein (CRP) values $<6 \mathrm{mg} /$ $\mathrm{L}$, indicating that no overt inflammation was present. Exclusion criteria applied to both patients with $\mathrm{SCZ}$ and healthy controls were: 1) use of supplements with antioxidants or $\omega 3$-polyunsaturated fatty acids the month prior to the study; 2) lifetime use of immunosuppressive drugs including glucocorticoids; 3 ) neurodegenerative and neuroinflammatory disorders including stroke, Parkinson's disease, multiple sclerosis, and Alzheimer's disease; and 4) (auto)immune illnesses including psoriasis, rheumatoid arthritis, chronic obstructive pulmonary disease (COPD), inflammatory bowel disease, and diabetes mellitus.

\section{Measurements}

\section{Clinical assessments}

A senior psychiatrist specialized in SCZ used a semistructured interview to assess sociodemographic and clinical data in patients and healthy controls. The psychiatrist made the diagnosis of SCZ based on DSM-IVTR criteria using the Mini-International Neuropsychiatric Interview (MINI) in a validated Arabic translation (Iraqi dialect). The same psychiatrist also assessed the Schedule for the Deficits Syndrome (SDS), ${ }^{24}$ the Scale for the Assessments of Negative Symptoms (SANS), ${ }^{25}$ the Brief Psychiatric Rating Scale (BPRS), ${ }^{26}$ the Hamilton Depression Rating Scale (HAM-D), ${ }^{27}$ and the Rating Scale for Fibromyalgia and Chronic Fatigue Syndrome (the FibroFatigue or FF scale). ${ }^{28}$ On the same day, a research psychologist, blinded to the clinical diagnosis, assessed neuropsychological probes using the Brief Assessment of Cognition in Schizophrenia (BACS), ${ }^{29}$ which covers the List Learning test (to assess verbal episodic memory), Digit Sequencing Task (to assess working memory), Category Instances (to assess semantic fluency), Controlled Word Association (to assess letter fluency), Symbol Coding (to assess attention), and Tower of London (to assess executive functions). We also assessed the drug state of the patients because 68 patients were treated with fluphenazine, 108 with risperidone, and 11 with olanzapine. The diagnosis of tobacco use disorder (TUD) was made using DSM-IV-TR criteria. Body mass index (BMI) was assessed the same day as the clinical interview and was scored as body weight $(\mathrm{kg}) /$ length $\left(\mathrm{m}^{2}\right)$.

\section{Assays}

After fasting ( $>8$ hours), venous blood $(5 \mathrm{~mL})$ was aspirated from patients and controls between 8:00 and 10:00 a.m. utilizing disposable needles and plastic syringes, and samples were transferred into a clean plain tube. Blood was left at room temperature for $15 \mathrm{~min}$ for clotting and centrifuged at 3,000 rpm for 10 minutes. Serum was then separated and stored in two Eppendorf at $-80^{\circ} \mathrm{C}$ for later analysis. Commercial ELISA sandwich kits were 
used to measure serum CCL11, IL-1 $\beta$, and TNF- $\alpha$ (Elabscience ${ }^{\circledR}$, Inc., Houston, USA). The sensitivities of the kits are $9.38 \mathrm{pg} / \mathrm{mL}$ for CCL11 kit, $4.69 \mathrm{pg} / \mathrm{mL}$ for IL-1 $\beta$ kit, and $4.69 \mathrm{pg} / \mathrm{mL}$ for TNF- $\alpha$ kit. All the measured concentrations were greater than the sensitivity of the assays. We did not apply left-censoring and used the measured concentration in the statistical analyses. The procedures were followed according to the manufacturer's instructions without modifications. The intra-assay coefficient of variation (CV) (precision within an assay) was $<6.22 \%$. Serum CRP was measured using a kit supplied by Spinreact ${ }^{\circledR}$ (Barcelona, Spain). The test is based on the principle of latex agglutination.

\section{Estimation of serum metals}

The concentrations of cesium, rubidium, and rhenium in the serum samples of patients with SCZ and controls were measured by using graphite furnace atomic absorption spectrophotometer (Shimadzu model GFA-6200) at the labs of the Ministry of Science and Technology/ Department of Research of Biochemical Science, Baghdad, Iraq. The measurements occurred at the specific wavelengths of each ultra-trace element. Standard solutions of the three metals were obtained from SigmaAldrich $^{\circledR}$ (Dermstadt, Germany) in a concentration of $1,000 \mu \mathrm{g} / \mathrm{L}$ each, and subsequent dilutions were carried out to obtain a calibration curve. All other reagents used were of atomic absorption spectroscopy (AAS) grade, and deionized water was used to ensure no leaching of any trace elements to the measured standard and samples. Small screw-capped polyethylene containers were used for the preparation of the samples, and all analyses were carried out as mentioned previously ${ }^{30}$ with some modifications. ${ }^{18}$ Briefly, the deep-frozen serum samples were reconstituted at room temperature and then centrifuged at $2,000 \mathrm{~g}$ for 15 minutes. Serum $(250 \mu \mathrm{L})$ was diluted into 1:8 (v/v) up to $2 \mathrm{~mL}$ with $0.5 \%(\mathrm{v} / \mathrm{v})$ nitric acid $\left(\mathrm{HNO}_{3}\right)$ for AAS measurements supplied by CPAChem Ltd, (Stara Zagora, Bulgaria) and internal standard solutions. The three metal element standards were mixed in the calibration solutions and diluted with the same nitric acid solution and internal standard solutions and then prepared in concentrations matching the concentrations of the trace elements in human serum. The certified reference mix (number 92091) used for internal quality control contains 33 elements at concentrations of $10 \mathrm{mg} / \mathrm{L}$ of $10 \% \mathrm{HNO}_{3}\left(\right.$ TraceCERT $^{\circledR}$, Sigma-Aldrich, Dermstadt, Germany) and was diluted according to product instructions and further prepared with $0.5 \% \mathrm{HNO}_{3}(\mathrm{v} / \mathrm{v})$. The percentage recovery of the measured metal concentrations was within the range of $90-98 \%$ of the certified values of the elements.

\section{Statistical analysis}

The differences in the scale variables, namely immune biomarkers and trace elements, were assessed using analysis of variance or the Mann-Whitney $U$ test (in case of heterogeneity of variance), while associations among two sets of categorical variables were assessed using analysis of contingency tables (chi-square $\left[\chi^{2}\right]$ tests). Correlations between scale variables were checked using Pearson's product moment correlations or partial correlations. Univariate and multivariate general linear model (GLM) analysis was used to examine the associations between explanatory variables and the three trace elements, while adjusting for age, sex, BMI, and drug state. Tests for between-subject effects were used to examine the associations between the significant explanatory variables and the elements. Multiple tests were p-corrected using the false discovery rate (FDR) method. All tests were two-tailed and a p-value of 0.05 was used for statistical significance. IBM SPSS version 25 for Windows was used to analyze the data.

\section{Ethics statement}

The study was conducted according to Iraq and international ethics and privacy laws. Written informed consent was obtained from all participants, as well as the firstdegree relatives of SCZ participants (the legally authorized representatives are father, mother, spouse, son, or brother) prior to participation in this study. Approval for the study was obtained from the ethics committee (institutional review board) of the College of Science, University of Kufa, Iraq (protocol n. 347/2019), which is in compliance with the International Guideline for Human Research protection as required by the Declaration of Helsinki.

\section{Results}

\section{Sociodemographic data}

Table 1 shows the sociodemographic data of participants with SCZ vs. healthy controls. There were no statistically significant differences in sex, TUD, marital status, residency, and BMI between patients with SCZ and normal controls. The number of unemployed participants was significantly higher in the SCZ group than in controls, while years of education were slightly lower in patients with SCZ. Patients with SCZ were somewhat older than controls. BPRS, SANS, FF, and HAM-D scores were significantly higher in patients than in controls. CCL11, IL$1 \beta$, and TNF- $\alpha$ were significantly higher in patients with SCZ as compared with healthy controls. All cognitive test scores were significantly lower in patients than controls.

\section{Intercorrelations}

In the overall study group, there were significant correlations between cesium and the total BPRS score $(r=$ -0.269, $p<0.001)$, SANS $(r=-0.247, p<0.001)$, FF scale $(r=-0.234, p=0.002)$ and the HAM-D $(r=-0.262$, $p<0.001$, all $n=174)$. Serum cesium levels were also correlated with the results of cognitive probes, namely Tower of London $(r=0.221, p=0.003)$, Symbol Coding $(r=0.276, p<0.001)$, COWA $(r=0.266, p<0.001)$, Category Instances $(r=0.19, p=0.012)$, Digit Sequencing Task $(r=0.225, p=0.003)$, and List Learning $(r=$ $0.195, p=0.010)$. In the SCZ sample, which is a restricted 
Table 1 Demographic, clinical, immune, and trace element data in healthy controls and patients with SCZ

\begin{tabular}{|c|c|c|c|c|c|}
\hline Variables & Control $(n=54)$ & $\operatorname{SCZ}(n=120)$ & $\mathrm{F} / \chi^{2}$ & df & $\mathrm{p}$-value \\
\hline Sex (female/male) & $18 / 36$ & $48 / 72$ & 0.703 & 1 & 0.402 \\
\hline TUD (yes/no) & $17 / 37$ & $42 / 78$ & 0.206 & 1 & 0.650 \\
\hline Marital status (married/single) & $23 / 31$ & $53 / 65$ & 0.081 & 1 & 0.776 \\
\hline Residency (urban/rural) & $52 / 2$ & $104 / 16$ & 3.723 & 1 & 0.054 \\
\hline Employment (yes/no) & $50 / 4$ & $22 / 98$ & 84.663 & 1 & $<0.001$ \\
\hline Income (US $\$$ /month) & $512.90(174.60)$ & $404.40(115.20)$ & 24.50 & $1 / 170$ & $<0.001$ \\
\hline Age (years) & $37.60 \pm 10.50$ & $41.00 \pm 9.70$ & 4.422 & $1 / 172$ & 0.037 \\
\hline BMI $\left(\mathrm{kg} / \mathrm{m}^{2}\right)$ & $26.90 \pm 3.83$ & $26.70 \pm 4.80$ & 0.066 & $1 / 172$ & 0.798 \\
\hline Education (years) & $14.30(4.90)$ & $12.30(4.20)$ & 7.599 & $1 / 172$ & 0.007 \\
\hline BPRS & $18.00 \pm 0.00$ & $63.70 \pm 14.00$ & MWU & - & $<0.001$ \\
\hline SANS & $1.04 \pm 0.60$ & $91.10 \pm 16.60$ & MWU & - & $<0.001$ \\
\hline HAM-D & $2.30(0.90)$ & $29.10(8.10)$ & MWU & - & $<0.001$ \\
\hline FibroFatigue scale & $1.10(0.90)$ & $23.90(4.30)$ & MWU & - & $<0.001$ \\
\hline $\mathrm{IL}-1 \beta(\mathrm{pg} / \mathrm{mL})$ & $0.75 \pm 0.30$ & $1.92 \pm 0.94$ & MWU & - & $<0.001$ \\
\hline TNF- $\alpha(\mathrm{pg} / \mathrm{mL})$ & $38.77 \pm 22.83$ & $60.44 \pm 14.12$ & MWU & - & $<0.001$ \\
\hline CCL11 (pg/mL) & $138.88 \pm 57.25$ & $281.59 \pm 81.64$ & MWU & - & $<0.001$ \\
\hline List Learning & $59.37 \pm 7.47$ & $25.65 \pm 9.94$ & MWU & - & $<0.001$ \\
\hline Digit Sequencing Task & $17.00 \pm 4.18$ & $4.32 \pm 2.95$ & MWU & - & $<0.001$ \\
\hline Category Instances & $70.28 \pm 6.25$ & $32.58 \pm 17.70$ & MWU & - & $<0.001$ \\
\hline COWA & $33.26 \pm 3.33$ & $6.17 \pm 4.05$ & MWU & - & $<0.001$ \\
\hline Symbol Coding & $79.39 \pm 7.84$ & $18.21 \pm 14.80$ & MWU & - & $<0.001$ \\
\hline Tower of London & $19.61 \pm 1.83$ & $6.32 \pm 4.54$ & MWU & - & $<0.001$ \\
\hline Rubidium ( $\mu \mathrm{g} / \mathrm{L}) *$ & $0.54 \pm 0.35$ & $0.65 \pm 0.38$ & 3.307 & $1 / 169$ & 0.071 \\
\hline Cesium $(\mu \mathrm{g} / \mathrm{L})^{*}$ & $1.14 \pm 0.46$ & $0.93 \pm 0.43$ & 8.819 & $1 / 169$ & 0.003 \\
\hline Rhenium $(\mu \mathrm{g} / \mathrm{L}) *$ & $0.04 \pm 0.02$ & $0.04 \pm 0.03$ & 0.511 & $1 / 169$ & 0.475 \\
\hline
\end{tabular}

Data presented as mean \pm standard deviation, unless otherwise specified and * except model-generated estimated marginal means (standard error) obtained by the univariate general linear model (GLM) analyses shown in Table 2.

$\mathrm{BMI}=$ body mass index; BPRS = Brief Psychiatric Rating Scale; CCL11 = eotaxin; COWA = Controlled Oral Word Association test; df = degrees of freedom; HAM-D = Hamilton Depression Rating Scale; IL = interleukin; MWU = Mann-Whitney $U$ test; SANS = Scale for the Assessment of Negative Symptoms; SCZ = schizophrenia; TNF = tumor necrosis factor; TUD = tobacco use disorder.

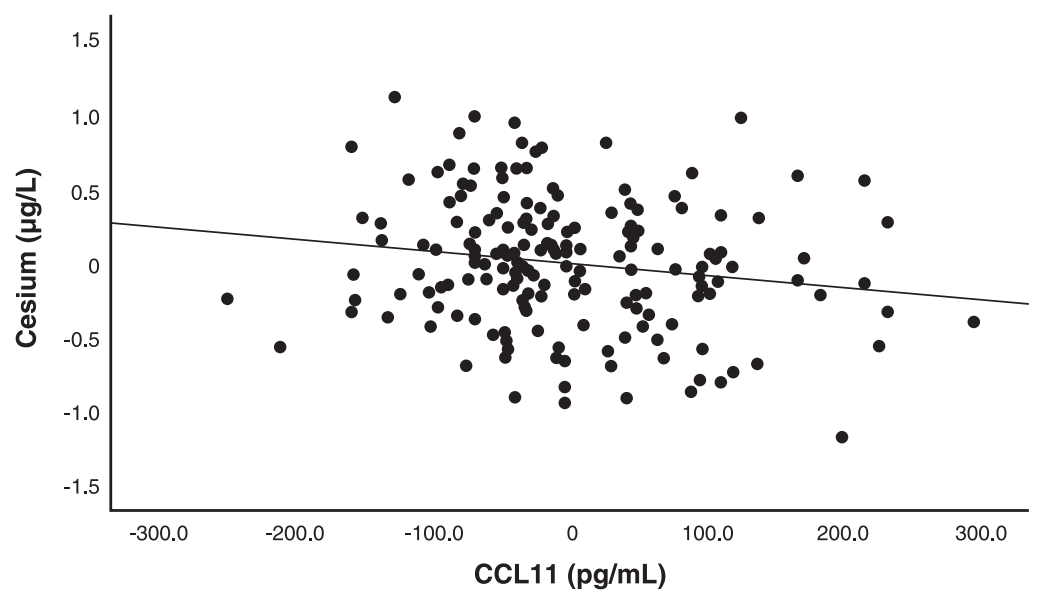

Figure 1 Partial regression plot of cesium on eotaxin (CCL11) levels showing a significant inverse association between both variables $(t=-2.21, p=0.028)$. Thus, this plot shows the correlation between Cs and CCL11 after controlling for the effects of age, sex, and BMI.

study sample, there was a significant association between cesium and Symbol Coding $(r=0.201, p=0.027, n=120)$. Moreover, we examined the associations between cesium and cognitive test results in the selected sample of participants with secondary school or graduate education and observed significant correlations between cesium and Tower of London $(r=0.191, p=0.038)$, Symbol Coding $(r=0.223, p=0.015)$, COWA $(r=0.218, p=$ $0.018)$, and Digit Sequencing Task $(r=0.215, p=0.019)$, while the other tests were significant using one-tailed tests. Cesium was significantly correlated with CCL11 $(r=-0.210, p=0.006)$ and TNF- $\alpha(r=-0.198, p=0.010)$, but not with IL-1 $\beta$ ( $r=-0.09, p=0.237)$. All the abovementioned significant associations remained significant after $\mathrm{p}$-correction for FDR and controlling for age, sex, and $\mathrm{BMI}$ in partial correlation analyses. Figure 1 shows the association (partial regression) between cesium levels and CCL11. There were no significant associations between the other trace elements and the immune biomarkers or clinical data. 
Table 2 Results of multivariate GLM analysis showing the associations between trace elements and diagnosis while adjusting for background variables

\begin{tabular}{|c|c|c|c|c|}
\hline Type of analysis*/dependent/explanatory variable & $\mathrm{F}$ & $\mathrm{df}$ & $p$-value & Partial $\eta^{2}$ \\
\hline \multicolumn{5}{|l|}{ Multivariate } \\
\hline \multicolumn{5}{|l|}{ Cesium, rubidium, rhenium } \\
\hline Diagnosis ${ }^{\dagger}$ & 4.466 & $3 / 167$ & 0.005 & 0.074 \\
\hline Sex & 0.876 & $3 / 167$ & 0.455 & 0.015 \\
\hline Age & 0.321 & $3 / 167$ & 0.810 & 0.006 \\
\hline $\mathrm{BMI}$ & 0.245 & $3 / 167$ & 0.865 & 0.004 \\
\hline \multicolumn{5}{|l|}{ Tests of between-subject effects } \\
\hline Cesium, diagnosis & 8.809 & 1 & 0.003 & 0.050 \\
\hline Rubidium, diagnosis & 3.307 & 1 & 0.071 & 0.019 \\
\hline Rhenium, diagnosis & 0.511 & 1 & 0.475 & 0.003 \\
\hline
\end{tabular}

$\mathrm{BMI}=$ body mass index; $\mathrm{df}=$ degrees of freedom; GLM = general linear model.

* Multivariate GLM was used to examine the differences in the three trace elements between patients and controls while covarying for sex, age and BMI. Test of between-subject effects or univariate GLM was performed to examine the differences in each of the three trace elements separately while covarying for sex, age and BMI.

Schizophrenia patients vs. healthy controls.

\section{Differences in trace elements between the study groups}

Table 2 displays the outcome of a multivariate GLM analysis comparing the three trace elements between the study groups while adjusting for age, BMI, and sex. There were significant differences in those elements between the study groups, with an effect size of 0.074 , while the covariates had no significant effects. Tests for betweensubject effects and Table 1, which shows the estimated marginal mean (standard error) values, indicate that cesium was significantly lower in patients with $\mathrm{SCZ}$ as compared with controls, and that the two other trace elements did not differ between the groups. The differences in cesium remained significant after $p$-correction $(p=0.009)$.

\section{Effects of background variables}

As shown above, age, sex, and BMI had no significant effects on serum trace element levels. TUD also had no significant effect on the measured trace elements $(F=0.66$, degree of freedom [df] $=3 / 166, p=0.576$, partial $\eta^{2}=0.012$ ). We also examined the possible effects of antipsychotic drug administration, and using multivariate GLM analysis and tests for between-subjects we found no significant effects of use of risperidone $\left(F=0.731, d f=3 / 166, p=0.535\right.$, partial $\left.\eta^{2}=0.013\right)$, olanzapine $\left(F=0.538, d f=3 / 166, p=0.657\right.$, partial $\eta^{2}=$ $0.010)$, and fluphenazine $(F=0.403$, df $=3 / 166, p=$ 0.732 , partial $\eta^{2}=0.008$ ) on the three metals even without $p$-correction for FDR. We also examined the effects of relevant sociodemographic and socioeconomic data (sex, age, marital status, TUD, urban/rural ratio, employment status, and income) on serum cesium levels and found that the differences between SCZ and controls remained significant $(F=6.24, d f=1 / 162, p=0.013)$ after covarying for these variables, which were all non-significant.

\section{Discussion}

The first major finding of this study is that serum cesium is significantly lowered in SCZ, while no significant changes in serum rhenium and rubidium were observed. These results extend those of Cai et al., ${ }^{15}$ who described a significant reduction in serum cesium in SCZ. As already noted, there is evidence that cesium shares similarities with potassium and competes with potassium for both active and passive membrane transport, ${ }^{31}$ although, after intake, cesium is distributed somewhat differently and exhibits a substantially longer whole-body retention time than potassium. ${ }^{31}$ Moreover, changes in the permeability of the brain-CSF barrier may occur in response to trace elements, including cesium, as observed in Alzheimer's disease. ${ }^{32}$ In this respect, Leggett et al. ${ }^{33}$ found that the plasma cesium/brain cesium ratio is 0.424 , indicating that cesium accumulates in the brain.

The second major finding of this study is the significant correlation among cesium levels and different symptom subdomains and cognitive impairments, including semantic memory, executive functions, spatial memory, episodic memory, and attention. These results further suggest that lowered cesium may contribute to the phenome of SCZ. ${ }^{6}$ Interestingly, in patients with Alzheimer's disease, cesium (and rubidium) concentrations were significantly lowered as compared with controls whilst plasma and cerebrospinal fluid cesium levels were strongly correlated. ${ }^{32}$ In CA1 hippocampal glial cells, cesium prevents maintenance of long-term depression, thereby affecting synaptic plasticity through blockade of potassium uptake. ${ }^{34}$ Moreover, cesium may also have some neuroprotective properties by inhibiting GSK3 $\beta$, preventing caspase- 3 activation and neuronal apoptosis, and attenuating peroxide-induced cell death. ${ }^{19}$ We therefore conclude that given its good blood-brain-barrier penetration, ${ }^{33}$ cesium may have some use in the treatment of neurodegenerative disorders.

The third major finding of our study is that the levels of cesium are significantly and inversely associated with immune biomarkers, namely CCL11 and TNF- $\alpha$, but not IL-1 $\beta$, although all three cytokines/chemokines are increased in SCZ. Interestingly, Cai et al. ${ }^{15}$ reported that the lowered cesium levels in SCZ are associated with lowered zinc and selenium concentrations. This is an important finding because lowered zinc is an immuneinflammatory biomarker associated with psychiatric 
diseases, including depression ${ }^{35}$ and $\mathrm{SCZ},{ }^{36}$ and because selenium has antioxidant and anti-inflammatory properties. ${ }^{37}$ Moreover, a previous study in rheumatoid arthritis reported a decrease in cesium, but not rubidium or rhenium ${ }^{18}$ suggesting that this metal profile is a response to activated immune-inflammatory pathways. Future research should examine whether lowered cesium could be the consequence of immune activation in SCZ or whether it plays a role in the neuroimmune pathophysiology of that disorder.

The present study has limitations that need to be addressed. Firstly, socioeconomic status (SES) can potentially interfere with the association between cesium and SCZ. One paper reported a strong association between the poverty income ratio (PIR) and changed levels in some trace elements/toxic metals, including higher cesium levels in high SES individual. ${ }^{38}$ Mediators of the association between PIR and selected trace elements/metals (including lead, cadmium, and mercury) comprised smoking, occupation, and diet (fish and shellfish), although no consistent mediators were found for the increased cesium levels. ${ }^{38}$ Nevertheless, in the current study, adjusting for possible effects of SES variables (including employment, income, urban/rural ratio, TUD, age, and sex) did not change the significant association between cesium and SCZ. Moreover, the present study did not include any individual with a PIR less than the Iraq poverty threshold, which is around 143.5 US $\$ /$ month, ${ }^{39}$ and all participants used a similar traditional Iraq diet. Secondly, while we adjusted our results for possible effects of the drug state of the patients, it is possible that lifetime exposure to antipsychotic agents may have contributed to the results. Nevertheless, similar immune disorders, which we found to be associated with lowered cesium, were also detected in drug-naïve patients with SCZ. ${ }^{4}$ Future research should replicate the cesium findings and adjust for lifetime exposure to different medications. Third, any associations between biomarkers and cognitive test results may, in theory, be affected by intervening variables, including education level. Nevertheless, the associations between cesium and cognitive probe results were also present in a restricted study sample including individuals with medium to higher levels of education.

In conclusion, serum cesium was decreased in SCZ, while no significant changes in rhenium and rubidium could be found. The lowered levels of cesium were associated with cognitive impairments, severity of SCZ symptoms domains, and inflammatory biomarkers (CCL11 and TNF- $\alpha$ ). These findings suggest that cesium may play a role in the pathophysiology of SCZ, for example by interference with potassium channels, immune-inflammatory pathways, lowered neuroprotection, or synaptic plasticity.

\section{Acknowledgements}

We are grateful to the staff of Ibn-Rushd hospital and especially Dr. Mokhlad Swadi Abed, for their help in the collection of samples. We also thank the high-skilled staff of Asia Clinical Laboratory in Najaf City for their help in the ELISA measurements, and the staff of the labs of the Ministry of Science and Technology, Department of Research of Biochemical Science, Baghdad, Iraq, for their measurements of ultra-trace elements levels.

\section{Disclosure}

The authors report no conflicts of interest.

\section{References}

1 Fleischhacker WW, Arango C, Arteel P, Barnes TR, Carpenter W, Duckworth K, et al. Schizophrenia--time to commit to policy change. Schizophr Bull. 2014;40 Suppl 3:S165-94.

2 Löhrs L, Hasan A. [Risk factors for the development of schizophrenia]. Fortschr Neurol Psychiatr. 2019;87:133-43.

3 Smith R, Maes M. The macrophage-T-lymphocyte theory of schizophrenia: additional evidence. Med Hypotheses. 1995;45:135-41.

4 Roomruangwong C, Noto C, Kanchanatawan B, Anderson G, Kubera $\mathrm{M}$, Carvalho AF, et al. The role of aberrations in the immuneinflammatory response system (IRS) and the compensatory immuneregulatory reflex system (CIRS) in different phenotypes of schizophrenia: the IRS-CIRS theory of schizophrenia. Mol Neurobiol. 2020;57:778-97.

5 Maes M, Kanchanatawan B, Sirivichayakul S, Carvalho AF. In schizophrenia, increased plasma IgM/IgA responses to gut commensal bacteria are associated with negative symptoms, neurocognitive impairments, and the deficit phenotype. Neurotox Res. 2019;35: 684-98.

6 Al-Hakeim HK, Almulla AF, Maes M. The neuroimmune and neurotoxic fingerprint of major neurocognitive psychosis or deficit schizophrenia: a supervised machine learning study. Neurotox Res. 2020;37: 753-71.

7 Yao JK, Keshavan MS. Antioxidants, redox signaling, and pathophysiology in schizophrenia: an integrative view. Antioxid Redox Signal. 2011;15:2011-35.

8 Zhang L, Pang B, Zhang W, Bai W, Yu W, Li Y, et al. Association between schizophrenia and DNA demethylase activity in human peripheral blood mononuclear cells. Clin Lab. 2018;64:1031-5.

9 Rodríguez B, Nani JV, Almeida PG, Brietzke E, Lee RS, Hayashi MA. Neuropeptides and oligopeptidases in schizophrenia. Neurosci Biobehav Rev. 2020;108:679-93.

10 Joe P, Getz M, Redman S, Kranz TM, Chao MV, Delaney S, et al. Transglutaminase-5 related schizophrenia. Schizophr Res. 2018;193: 477-9.

11 Saghazadeh A, Mahmoudi M, Shahrokhi S, Mojarrad M, Dastmardi $M$, Mirbeyk $M$, et al. Trace elements in schizophrenia: a systematic review and meta-analysis of 39 studies ( $N=5151$ participants). Nutr Rev. 2020;78:278-303.

12 Cao B, Yan L, Ma J, Jin M, Park C, Nozari Y, et al. Comparison of serum essential trace metals between patients with schizophrenia and healthy controls. J Trace Elem Med Biol. 2019;51:79-85.

13 Li Z, Liu Y, Li X, Ju W, Wu G, Yang X, et al. Association of elements with schizophrenia and intervention of selenium supplements. Biol Trace Elem Res. 2018;183:16-21.

14 Chen X, Li Y, Zhang T, Yao Y, Shen C, Xue Y. Association of serum trace elements with schizophrenia and effects of antipsychotic treatment. Biol Trace Elem Res. 2018;181:22-30.

15 Cai L, Chen T, Yang J, Zhou K, Yan X, Chen W, et al. Serum trace element differences between schizophrenia patients and controls in the Han Chinese population. Sci Rep. 2015;5:15013.

16 Horn S, Naidus E, Alper SL, Danziger J. Cesium-associated hypokalemia successfully treated with amiloride. Clin Kidney J. 2015;8: 335-8.

17 Hashimoto R, Ohi K, Yasuda Y, Fukumoto M, Yamamori $\mathrm{H}$, Kamino $\mathrm{K}$, et al. The $\mathrm{KCNH} 2$ gene is associated with neurocognition and the risk of schizophrenia. World J Biol Psychiatry. 2013;14:114-20.

18 Al-Hakeim HK, Moustafa SR, Jasem KM. Serum cesium, rhenium, and rubidium in rheumatoid arthritis patients. Biol Trace Elem Res. 2019;189:379-86.

19 Zhong J, Yao W, Lee W. Cesium chloride protects cerebellar granule neurons from apoptosis induced by low potassium. Int $\mathrm{J}$ Dev Neurosci. 2007;25:359-65. 
20 Wilkinson SM, Gunosewoyo H, Barron ML, Boucher A, McDonnell M, Turner $\mathrm{P}$, et al. The first CNS-active carborane: a novel P2X7 receptor antagonist with antidepressant activity. ACS Chem Neurosci. 2014;5:335-9.

21 Relman AS. The physiological behavior of rubidium and cesium in relation to that of potassium. Yale J Biol Med. 1956;29:248-62.

22 von Scholten BJ, Hasbak P, Christensen TE, Ghotbi AA, Kjaer A, Rossing $\mathrm{P}$, et al. Cardiac $82 \mathrm{Rb} \mathrm{PET} / \mathrm{CT}$ for fast and non-invasive assessment of microvascular function and structure in asymptomatic patients with type 2 diabetes. Diabetologia. 2016;59:371-8.

23 Rhenium: biological information [Internet]. 2020 Apr [cited 2020 Apr 29]. www.webelements.com/rhenium/biology.html

24 Kirkpatrick B, Buchanan RW, McKenny PD, Alphs LD, Carpenter WT Jr. The schedule for the deficit syndrome: an instrument for research in schizophrenia. Psychiatry Res. 1989;30:119-23.

25 Andreasen NC. The scale for the assessment of negative symptoms (SANS): conceptual and theoretical foundations. $\mathrm{Br} \mathrm{J}$ Psychiatry Suppl. 1989;(7): 49-52.

26 Overall JE, Gorham DR. The brief psychiatric rating scale. Psychol Rep. 1962;10:799-812.

27 Hamilton M. A rating scale for depression. J Neurol Neurosurg Psychiatry. 1960;23:56-62.

28 Zachrisson O, Regland B, Jahreskog M, Kron M, Gottfries CG. A rating scale for fibromyalgia and chronic fatigue syndrome (the FibroFatigue scale). J Psychosom Res. 2002;52:501-9.

29 Keefe RS, Goldberg TE, Harvey PD, Gold JM, Poe MP, Coughenour $\mathrm{L}$. the brief assessment of cognition in schizophrenia: reliability, sensitivity, and comparison with a standard neurocognitive battery. Schizophr Res. 2004;68:283-97.

30 Liu X, Piao J, Huang Z, Zhang SQ, Li W, Tian Y, et al. Determination of 16 selected trace elements in children plasma from China economical developed rural areas using high resolution magnetic sector inductively coupled mass spectrometry. J Anal Methods Chem. 2014;2014:975820.

31 Paquet F, Etherington G, Bailey M, Leggett RW, Lipsztein J, Bolch W, et al. ICRP Publication 130: Occupational intakes of radionuclides: Part 1. Ann ICRP. 2015;44:5-188.

32 Gerhardsson L, Lundh T, Minthon L, Londos E. Metal concentrations in plasma and cerebrospinal fluid in patients with Alzheimer's disease. Dement Geriatr Cogn Disord. 2008;25:508-15.

33 Leggett RW, Williams LR, Melo DR, Lipsztein JL. A physiologically based biokinetic model for cesium in the human body. Sci Total Environ. 2003;317:235-55.

34 Janigro D, Gasparini S, D'Ambrosio R, McKhann G, DiFrancesco D. Reduction of $\mathrm{K}^{+}$uptake in glia prevents long-term depression maintenance and causes epileptiform activity. J Neurosci. 1997;17: 2813-24.

35 Twayej AJ, Al-Hakeim HK, Al-Dujaili AH, Maes M. Lowered zinc and copper levels in drug-naïve patients with major depression: effects of antidepressants, ketoprofen and immune activation. World J Biol Psychiatry. 2020;21:127-38.

36 Joe P, Petrilli M, Malaspina D, Weissman J. Zinc in schizophrenia: a meta-analysis. Gen Hosp Psychiatry. 2018;53:19-24.

37 Solovyev ND. Importance of selenium and selenoprotein for brain function: from antioxidant protection to neuronal signalling. J Inorg Biochem. 2015;153:1-12.

38 Tyrrell J, Melzer D, Henley W, Galloway TS, Osborne NJ. Associations between socioeconomic status and environmental toxicant concentrations in adults in the USA: NHANES 2001-2010. Environ Int. 2013;59:328-35.

39 Ameen JR. Optimal multi-dimensional poverty lines: the state of poverty in Iraq. AIP Conf Proc. 2017;1872:020003. 